\title{
Redensificación y cooperativismo como instrumentos para la regeneración urbana sostenible de barrios vulnerables en Madrid
}

\author{
Francisco Javier González González ${ }^{1}$, Susana Moreno Soriano² ${ }^{2}$ José \\ Miguel Márquez Martinón ${ }^{3}$ \\ ${ }^{12}$ Departamento de Arquitectura, Universidad Europea, Madrid, España, \\ ${ }^{3}$ Grupo de Investigación ELAN. Universidad Europea, Madrid, España. \\ E-mail: ' franciscojavier.gonzalez@universidadeuropea.es, \\ 22susana.moreno@universidadeuropea.es, ${ }^{3}$ marquezmartinon@gmail.com
}

\begin{abstract}
Resumen. Hasta la crisis financiera de 2008, la regeneración urbana en barrios vulnerables de Madrid se basaba en la inversión pública, que asumía entre el 60 y el $70 \%$ de los costes de rehabilitación de las viviendas. A partir de 2008, esta inversión pública se retrae y la población se empobrece. En estas circunstancias, un modo de hacer viable estas intervenciones es utilizar recursos económicos locales, implícitos en el propio proceso de regeneración. Entre estos recursos se encuentra el aumento de edificabilidad, tradicionalmente asociado a modos de producción expansiva de la ciudad y a su planificación. Ahora bien, la redensificación ha de ser utilizada respondiendo a nuevos criterios: ¿cuál es el crecimiento mínimo necesario para hacer viable la regeneración urbana sostenible?, ¿cómo evaluar que estemos ante un proceso desarrollado con criterios de sostenibilidad?, y, por último, ¿cómo asegurar que la plusvalía generada se utiliza en la rehabilitación de las viviendas existentes?

Este artículo presenta los resultados de una investigación que pretende responder a estas cuestiones, tomando como caso el barrio del Aeropuerto en Madrid. Se han propuesto tres escenarios de intervención que implican incrementos de la edificabilidad, considerando las necesidades de rehabilitación energética de las viviendas existentes, y se han evaluado con indicadores de sostenibilidad urbana, fácilmente aplicables desde la administración local. De todo ello se deduce que la redensificación puede ser parte de una estrategia sostenible. Por último, se discute la necesidad de gestión público-privada considerando las cooperativas vecinales de regeneración urbana para redistribuir los beneficios producto de la redensificación
\end{abstract}

Palabras clave: Redensificación, cooperativas, regeneración urbana sostenible, indicadores sostenibilidad urbana, viabilidad económica

Introducción. ¿qué sentido tiene la redensificación en la regeneración urbana de la periferia madrileña?

La Máquina de Crecimiento Urbano (MCU) en Madrid, ha permitido la expansión por el territorio hasta llegar a conformar una región metropolitana de más de seis millones de habitantes. (De Santiago, 2011; Fernández y Roch, F. 2012, Roch, F. 2004 y 2000; Naredo J.M. 2004). Este potente artefacto económico e institucional ha tenido su expresión más intensa desde mediados de los años 90 del siglo pasado hasta su desmantelamiento (itemporal?) a partir de la crisis financiera mundial de 2008, y que, por motivos en los que nos vamos a entrar, 
pone a temblar a todo un sector económico como es el de la construcción. Antes de que esto ocurra, en Madrid ciudad se viven momentos de actividad inmobiliaria exuberante a la altura de los primeros 2000, y que coinciden con una gran afluencia de ingresos canalizados por la administración local hacia la inversión pública en áreas de regeneración urbana.

En efecto, las ARI (áreas de rehabilitación integral), gestionadas entre 1994 y 2012, suponen un instrumento eficaz para mejorar el estado de la edificación, actualizar el espacio público, sumar dotaciones donde nos las hay $\mathrm{y}$, en algunos casos, hasta atender directamente los problemas sociales emergentes (envejecimiento, nuevas culturas que traen consigo los inmigrantes, etc.), así como trabajar en una mayor cohesión entre vecinos. Las cifras que arroja esta actividad son llamativas: el esfuerzo rehabilitador realizado y previsto desde 1994 atañe a aproximadamente 111.000 viviendas (González y Muinelo 2013). Una de las claves de este tipo de procesos es la subvención pública en la rehabilitación de vivienda privada, de tal modo que en estas áreas los planes de vivienda han pagado hasta el $70 \%$ del coste de las obras para mejora de la habitabilidad, corriendo a cargo del propietario de cada vivienda el resto. Nos encontramos, por tanto, ante una situación de redistribución hacia la regeneración urbana de la plusvalía generada por la MCU que es liderada por la administración pública.

A riesgo de simplificar, con el aterrizaje de la crisis financiera de 2008 en las ciudades, se corta la inversión de las administraciones, también las locales. Además, se pone en grandes dificultades de supervivencia a un sector productivo, el de la construcción, que, históricamente enfocado en la producción de vivienda nueva, pasa de tramitar más de 250.000 visados en 2.007 a tan solo 50.000 en 2009. (BBVA 2008). La reacción ante la debacle pasa por hacer girar el sector hacia la rehabilitación de edificios, una actividad que hasta el momento no ha ocupado más que un lugar residual entre los empresarios, a pesar de las políticas urbanas descritas. Para ello la administración central se propone hacer desaparecer las barreras históricas que lastraban la rehabilitación con la ley 8/2013 de
Rehabilitación, Regeneración y Renovación Urbanas y un plan de vivienda que pretende impulsar el alquiler. En ella se le da especial relevancia a la rehabilitación energética de edificios y a la capitalización de los potenciales ahorros como medio de asumir los costes de la rehabilitación privada y sustituir las inversiones públicas del pasado.(Cuchi y Sweatman 2010), (Moreno, Susana., González, Francisco J. y Gómez Alberto.2014). Si atendemos a las escasas viviendas rehabilitadas desde 2013 es evidente que las medidas no han tenido el éxito esperado y parece oportuno repensar los medios de financiación de la regeneración de barrios.

En estas circunstancias, un modo de hacer viable estas intervenciones es utilizar recursos económicos locales, implícitos en el propio proceso de regeneración. Entre estos recursos se encuentra el aumento de edificabilidad, tradicionalmente asociado a modos de producción expansiva de la ciudad y a su planificación. En definitiva, como ya ocurría con la MCU, lo que se trata es de generar plusvalía en el proceso de transformación de la ciudad, consistiendo ahora esa transformación en la mejora de los barrios existentes y no en un crecimiento disfuncional que consume territorio reduciendo la sostenibilidad del sistema a largo plazo. ¿Pero, en qué condiciones se ha de generar esa plusvalía? La redensificación ha de ser utilizada respondiendo a nuevos criterios: ¿cuál es el crecimiento mínimo necesario para hacer viable la regeneración urbana sostenible?, ¿cómo evaluar que estemos ante un proceso desarrollado con criterios de sostenibilidad?, y, por último, ¿cómo asegurar que la plusvalía generada se utiliza en la rehabilitación de las viviendas existentes?

El presente artículo expone los resultados de una investigación cuyo objetivo es discutir en un barrio de la periferia madrileña, que instrumentos se pueden implementar para controlar que una operación de redensificación no se escape de un marco de sostenibilidad ambiental y que es capaz de producir recursos para asumir el coste de la rehabilitación de los edificios existentes. 
Hacia un instrumento metodológico para evaluar la redensificación, en el marco de la sostenibilidad urbana madrileña

Que la densificación puede ser un instrumento para mejorar la sostenibilidad de ciudades y estructuras urbanas de baja densidad es algo incorporado a la cultura urbanística desde los primeros informes de (Newman y Kenworthy, 1989) sobre consumo de combustible y densidad urbana. De lo que se trata aquí es de saber cuales son los límites a la redensificación en estructuras urbanas medianamente densas sin comprometer la sostenibilidad del conjunto de la ciudad. Para ello el primer paso de esta metodología supone establecer qué tipo de actuaciones describen una intervención de regeneración urbana sostenible (RUS) y cómo se puede expresar a través de indicadores que permitan evaluar una actuación de forma sencilla .

Para ello se han cruzado tres fuentes.

a) La primera es la elección de un sistema de indicadores. Para ello se parte del Sistema de indicadores y condicionantes para ciudades grandes y medianas. IV Reunión del Grupo de trabajo de Indicadores de Sostenibilidad de la Red de Redes de Desarrollo Local Sostenible que es el instrumento de medida habitual del ayuntamiento de Madrid. Se han filtrado y reelaborado estos indicadores para ajustarlos a la escala de barrio de las actuaciones de regeneración.

b) En segundo lugar, se han analizado 52 actuaciones pertenecientes al programa europeo CONCERTO para el intercambio y financiación parcial de experiencias de transformación urbana de diferentes escalas con el eje común eficiencia energética y la producción local de energías renovables. El objetivo de este análisis es identificar si los indicadores son aplicables a estos ejemplos, teniendo en consideración los tipos de actuaciones que se incluyen en ellos, con el fin de deducir los "ingredientes" más habituales a incluir en un proceso de regeneración en la periferia madrileña. También se han utilizado algunos ejemplos recogidos en bibliografía especializada para completar la visión. (Claassens, Koomen, and Rijken Bart 2018) (Boorsboom-Van Beurden, Doepel, and Tillie,
2013)

c) En tercer lugar, se ha realizado una identificación análoga de objetivos y temas en el consenso institucional, actualizado en los últimos años en la Declaración de Toledo .

Con esta batería de indicadores revisada se pretende cumplir varios objetivos: hacer descender los indicadores municipales actuales en Madrid a la escala de barrio, que se puedan manejar como indicadores de estado, esto es, que permitan diagnosticar una situación previa a la intervención, y que permitan medir impactos y estrategias, por ejemplo, la del aumento de la edificabilidad en un barrio. (Ver figura 1)

El procedimiento para utilizar estos indicadores incluye el acompañamiento de análisis específicos sobre la situación de partida, tales como la cuantificación de la demanda energética de la edificación del barrio antes de ser regenerado. Indicadores y análisis son el punto de partida para establecer estrategias de intervención en diferentes escenarios. Finalmente, los indicadores permiten evaluar su impacto sobre la sostenibilidad comparándolo con la situación original y discutiendo que variables empeoran y cuales mejoran en cada uno de dichos escenarios. (Ver figura2). Veamos los escenarios posibles para el caso del Barrio del Aeropuerto.

El barrio del Aeropuerto en Madrid. Escenarios para la redensificación en una operación de regeneración urbana sostenible.

El barrio del aeropuerto en Madrid se encuentra en el noreste de Madrid, en el distrito de Barajas y está delimitado, por un lado, por las carreteras A2 y M11, que conecta la M40 y el aeropuerto de Barajas, y su tercer límite es la terminal de carga del aeropuerto y los desarrollos logísticos adyacentes.

La morfología del barrio es típica de los desarrollos residenciales de los años 50 y 60 en la periferia de las ciudades españolas. Edificios de tres plantas, en su inmensa mayoría sin ascensor, que albergan 568 viviendas, de las cuales el $45 \%$ son viviendas pasantes, con doble orientación. El estado de la edificación reviste carencias importantes, más allá de la 
falta de accesibilidad vertical o las dificultades de conseguir condiciones de confort a través de sistemas pasivos. La envolvente es muy poco eficiente desde el punto de vista energético y las patologías derivadas del alto nivel freático, las inundaciones de algunos puntos del espacio público y las limitaciones constructivas conllevan problemas de humedad en casi todos

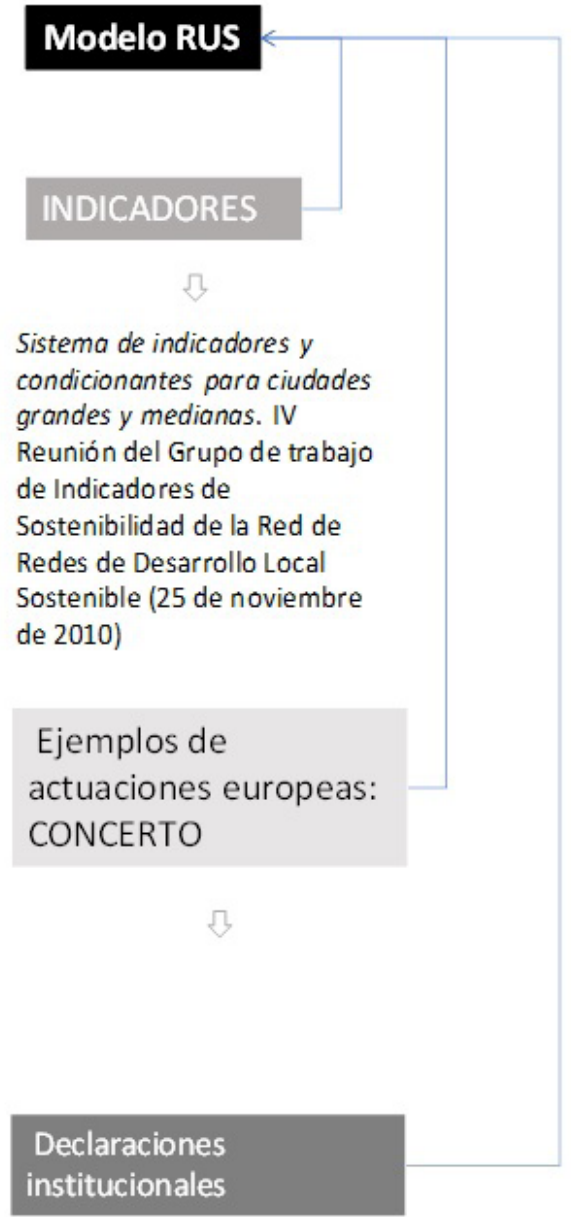

Declaración de Toledo 2010

\section{Modelo RUS. Definido a través de estrategias}

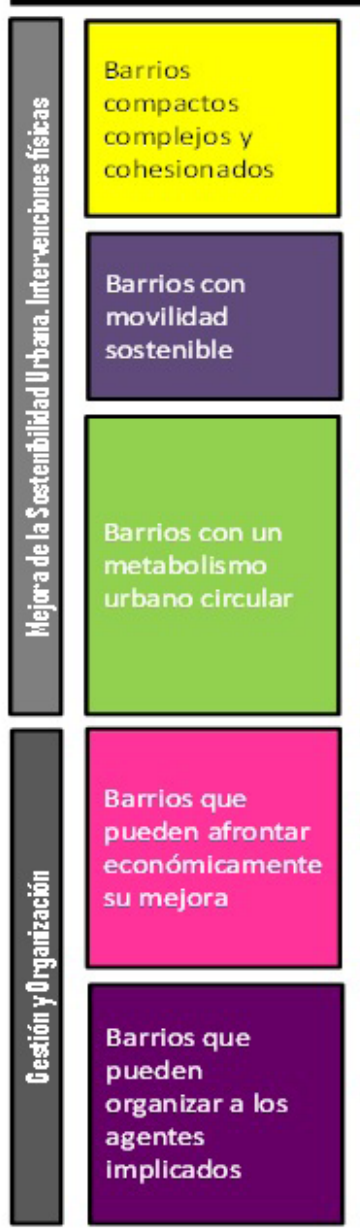

E1. de compacidad, y diversificación funcional

E2. de mejora de la habitabilidad básica

E3. Movilidad sostenible centrada en el peatón, la estancialidad y los modos verdes.

E4. sobre la energía
E5. sobre el agua

E6. sobre los materiales y residuos

E7. sobre la naturalización

E8. sobre la obtención de recursos locales

E9. sobre la obtención de
financiación, inversión,
subvención

E10. sobre mecanismos de colaboración público-privada

E11. sobre vinculación de los vecinos y la generación de capital social

Fig. 1. Metodología para definir un modelo de Regeneración Urbana Sostenible expresado a través de estrategias e indicadores. Fuente propia

los edificios que comprometen las condiciones de habitabilidad de muchas viviendas. El espacio público se ve muy presionado por las necesidades de aparcamiento de los trabajadores de las oficinas circundantes lo que compromete una coherencia clara en la estructura y en el uso social de espacios libres de uso público.

Se establece una serie de estrategias de mejora ambiental y de la habitabilidad de los edificios existentes que son comunes a los tres escenarios con los que vamos a trabajar: El aislamiento de la envolvente y la instalación de los ascensores, junto con la solución de patologías derivadas de las humedades y la mejora de los mecanismos pasivos de una rehabilitación bioclimática posible son los retos que corresponden a la mejora de la edificación. La mejora del metabolismo urbano del barrio está asociada al reforzamiento de la cohesión social, y pasa por reconfigurar el espacio público y dar solución a los problemas de 


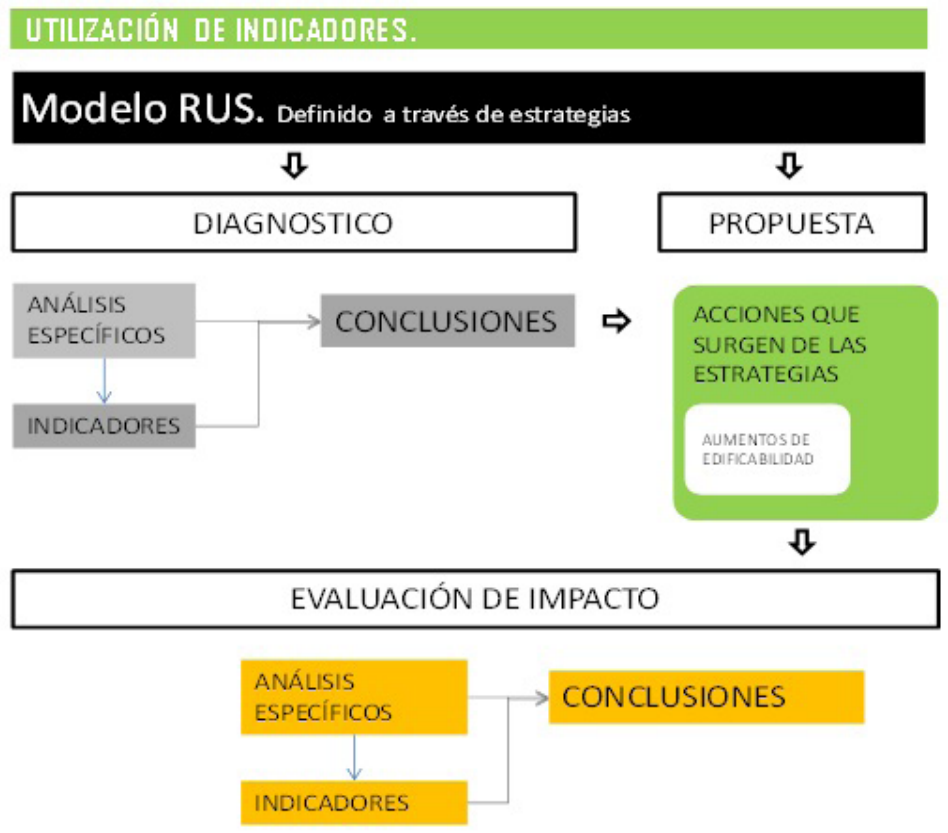

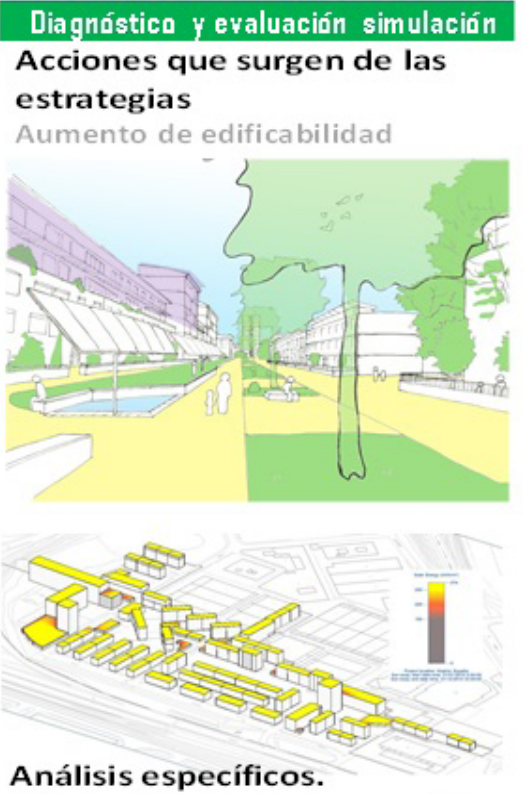

Cálculo de la demanda energética.

Fig. 2. Análisis específicos e indicadores para evaluar el impacto de las estrategias de regeneración. Fuente propia

inundaciones, a recuperar espacio de relación social al disminuir la presión del vehículo privado y activar las conexiones peatonales con otros barrios.
En la estrategia de regeneración urbana se han utilizado tres escenarios que suponen crecimientos análogos pero que suponen estrategias para ubicar la nueva edificabilidad
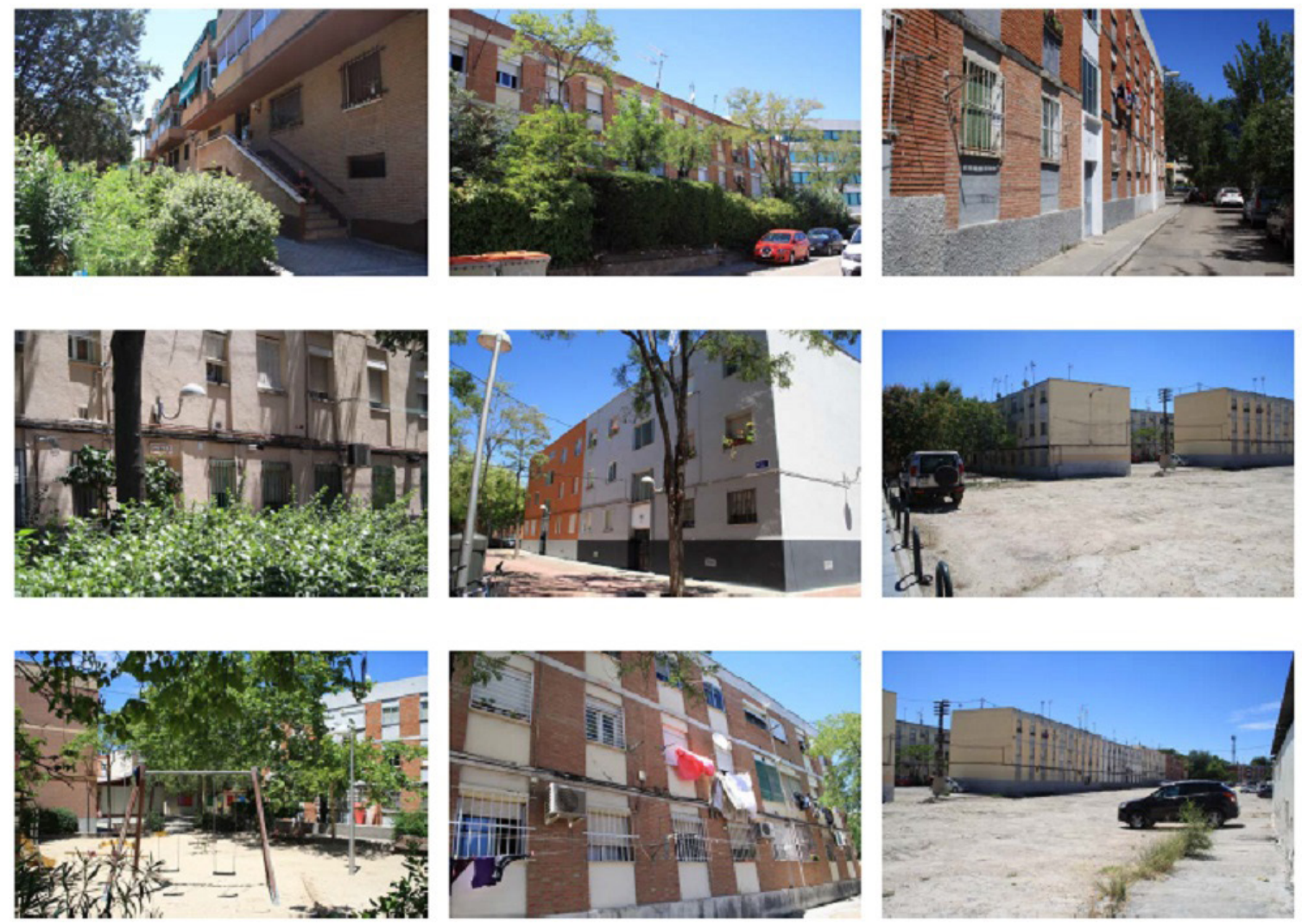

Fig. 3. Imágenes del estado actual del barrio del Aeropuerto. Fuente propia 
bien diferenciadas. En ellos se evalúa el impacto de los incrementos de edificabilidad:

a) Escenario 1. En él se pasa de 568 viviendas a 882 , situadas en algunos edificios de los existentes, aumentando el número de plantas de 3 a 5 y sumando equipamientos que completan las necesidades dotacionales del barrio, a la vez que abre la posibilidad de que parte de la edificabilidad se dedique a usos no residenciales, con el fin de incrementar la diversidad funcional del barrio. En este escenario la edificabilidad añadida supone, por un lado, un incremento de alturas en los edificios con viviendas que son pasantes, en las cuales es más sencillo instalar el ascensor y por tanto crecer en altura, y por otro, en edificios de nueva planta. Los crecimientos en altura de edificios existentes son de dos nuevas plantas.

b) Escenario 2. Es un escenario semejante al primero. Se pasa de 568 viviendas a 864, en este caso ubicadas en la mayor parte de los edificios existentes subiendo siempre un piso más y también en edificios de nueva planta. Las condiciones de mezcla de usos que supone el escenario son iguales a las del anterior.

c) Escenario 3. El incremento de viviendas en este caso supone llegar hasta las 826 y siempre en edificios de nueva planta.

\section{¿Es sostenible la redensificación del barrio? Evaluación de la sostenibilidad ambiental de los escenarios propuestos}

A la vista de los tres escenarios caben dos preguntas ¿son escenarios sostenibles en lo ambiental y en lo social? ¿y cuál de ellos lo es más? Podemos establecer algunas consideraciones:

Aplicando la batería de indicadores se comprueba que en los treinta indicadores empleados los valores mejoran en todos ellos. Sin embargo, en la situación actual sólo lo cumplen 14 de ellos y en todos los escenarios el número de indicadores por encima del valor de referencia varían entre 23 y 25 según los escenarios. (entre un 76 y un $83 \%$ ).

Los indicadores relativos a barrios compactos y complejos mejoran claramente en los tres

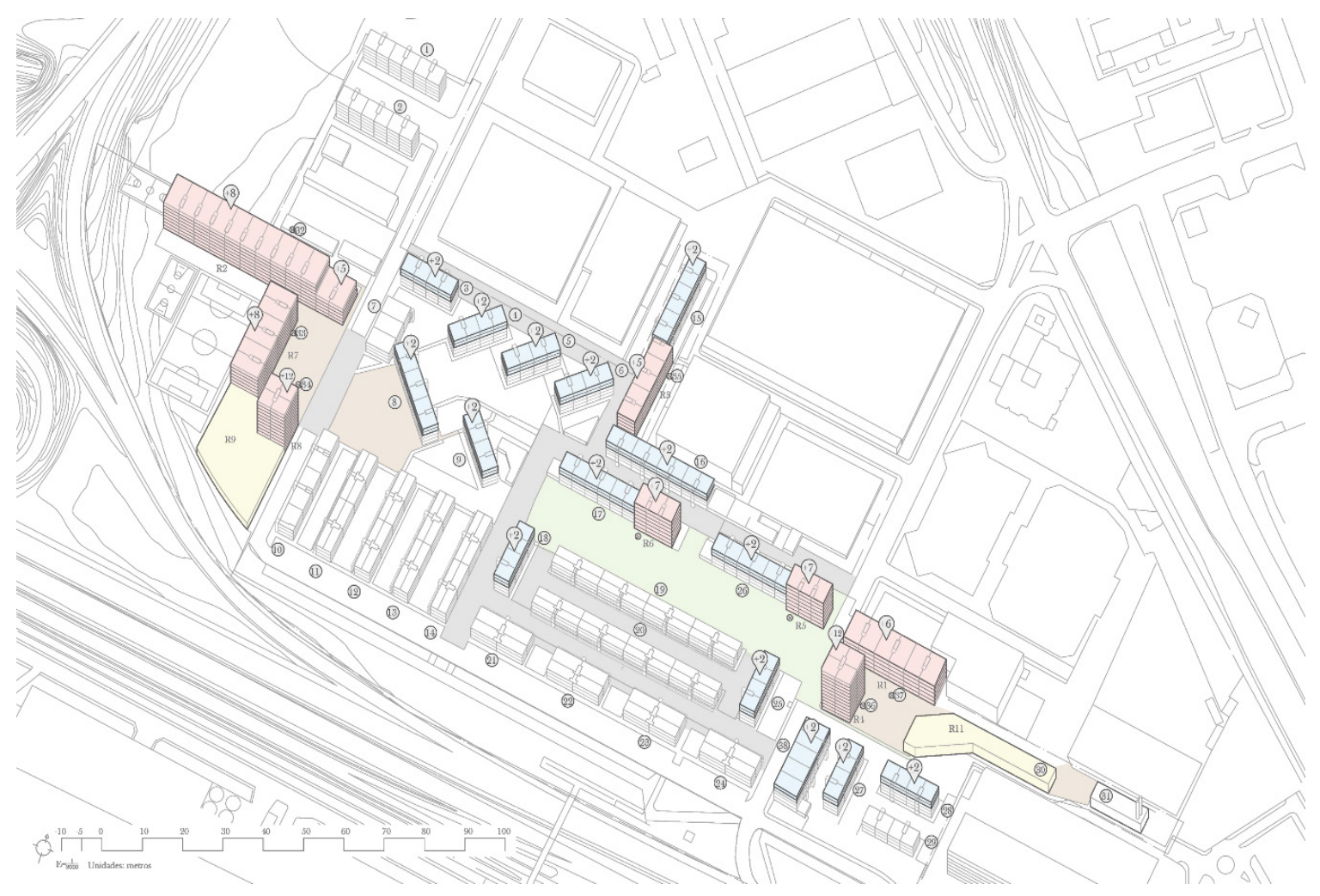

Fig. 4. Escenario 1 Volumetría. Azul plantas recrecidas sobre edificios existentes. Rosa edificios nueva planta, amarillo nuevos edificios de equipamiento 


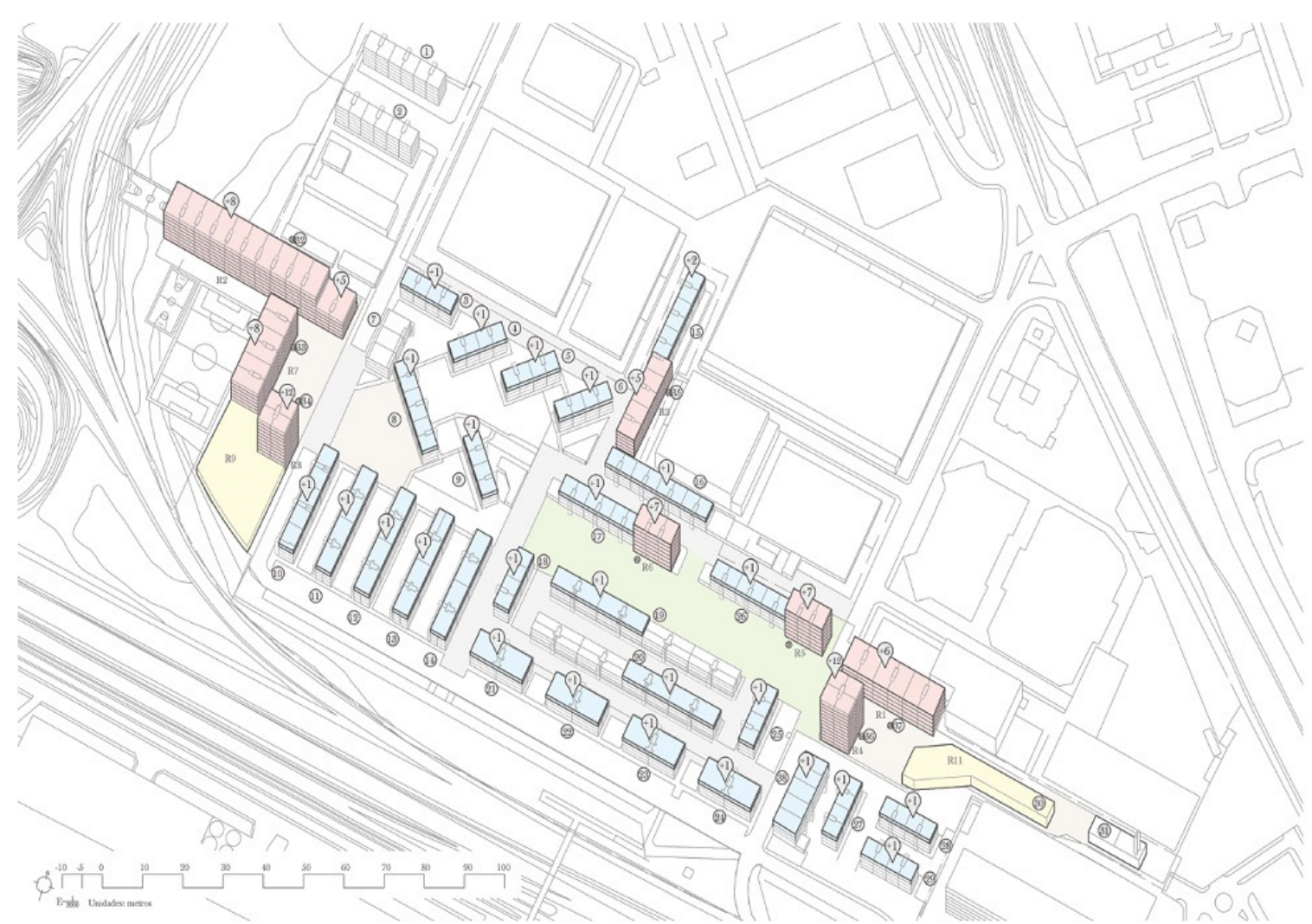

Fig. 5. Escenario 2 Volumetría. Azul plantas recrecidas sobre edificios existentes. Rosa edificios nueva planta, amarillo nuevos edificios de equipamiento. Fuente propia

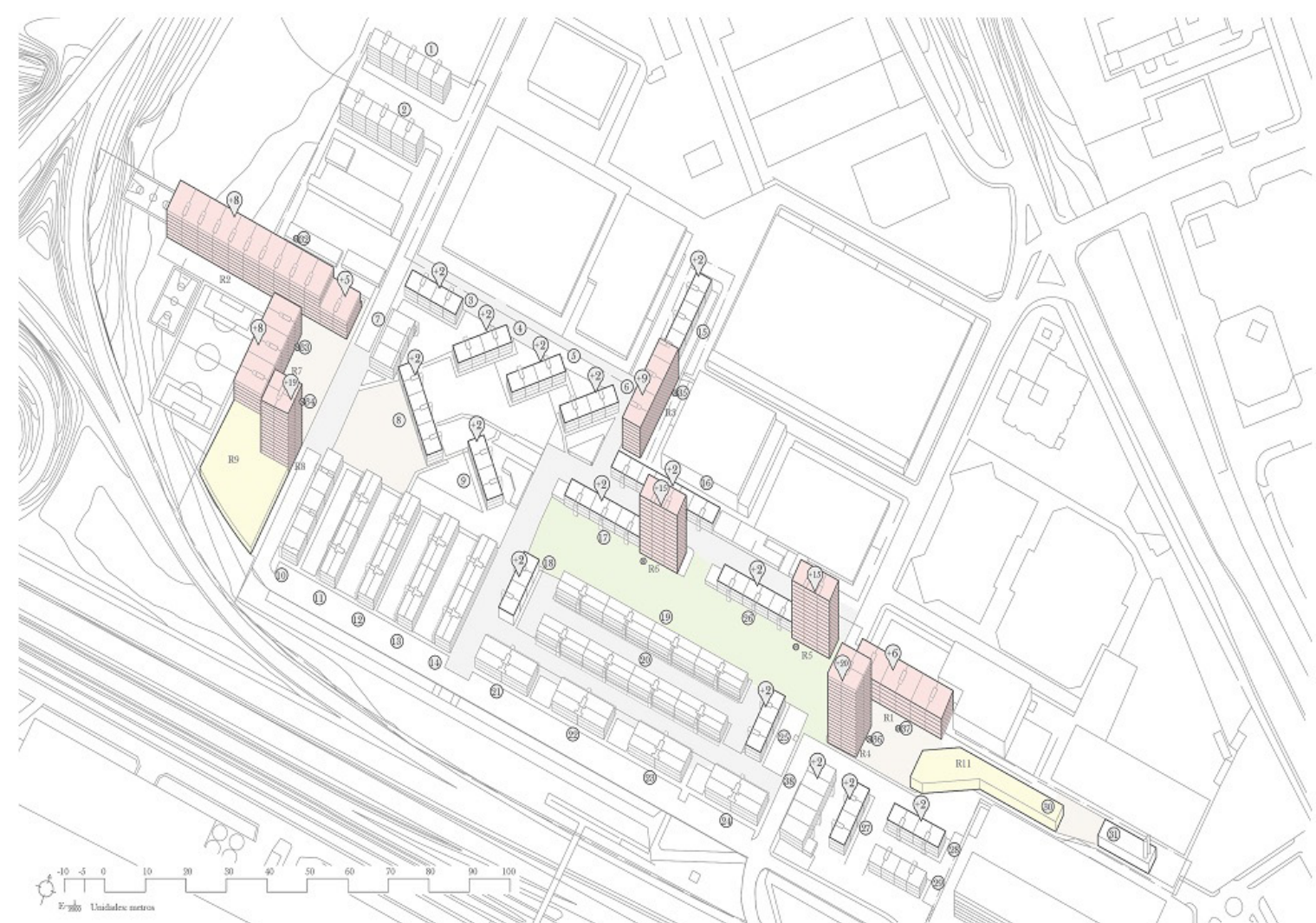

Fig. 6. Escenario 3 Volumetría. Rosa edificios nueva planta, amarillo nuevos edificios de equipamiento. Fuente propia 
escenarios. si bien sólo se cumplen finalmente el $80 \%$ de los mismos. Así, sobre una densidad de partida fuera de la horquilla óptima de entre 70 y 100 has, los escenarios exploran al máximo las posibilidades de redensificación del barrio. Esto implica que sigue siendo un barrio con una relativa compacidad baja, a pesar de casi duplicar la edificabilidad existente. En la regeneración urbana. Esto permite la existencia de mucho espacio entre las edificaciones.

Sobre los indicadores relativos a la movilidad sostenible y las condiciones de habitabilidad del espacio público en el barrio se comprueba que todos los indicadores mejoran y alcanzan valores aceptables en este bloque, exceptuando el relativo al acceso a las infraestructuras para la bici, ya que no hay carriles bici cercanos. La bici es compatible en coexistencia con el resto de los modos de movilidad dentro del barrio. La proporción de espacio para el peatón se invierte completamente con las medidas de reurbanización de las calles que se proponen. Así, de poco más de un $45 \%$ de espacio para el peatón a poco más de un $75 \%$, considerando las plazas y las aceras y excluyendo las zonas verdes.

Sobre los indicadores relativos al metabolismo urbano y la biodiversidad podemos decir que se cumplen los indicadores en el $77 \%$ de los casos. Los cambios entre escenarios se dan tan solo en el indicador relativo a la potencial eficiencia energética de las viviendas basadas en la radiación que reciben. En muchos casos, los indicadores no dan valores aceptables en la situación actual del barrio sencillamente porque no existen servicios o infraestructuras relativas a la mejora de su metabolismo (huertos urbanos, producción energética, etc.), sin embargo, sí hay espacio suficiente para implementar estos procesos en la simulación. Si nos fijamos en un indicador clave, el impacto del aumento de volumen sobre el soleamiento de las viviendas existentes, se comprueba que para los tres escenarios se consigue tener soleadas hasta un $80 \%$ de las viviendas existentes. Por otro lado, en el escenario 1, la proporción entre las viviendas afectadas y las que finalmente tiene estas condiciones de soleamiento es el más equilibrado, y también es aquel en que menos viviendas se ven perjudicadas, tan sólo 29 .

Por su parte, los análisis de demanda energética señalan que el escenario 1 es el más favorable y en él se obtendría un consumo promedio de energía final de $48,88 \mathrm{kWh} /$ m2año y de $62,96 \mathrm{kWh} / \mathrm{m} 2$ año en energía primaria. Esto significa que se ha reducido el consumo con respecto al barrio inicial en tanto en energía primaria como en final en torno a un $65 \%$. En lo que se refiere al análisis de la huella de carbono de la edificación del barrio rehabilitado con aumento de edificabilidad,

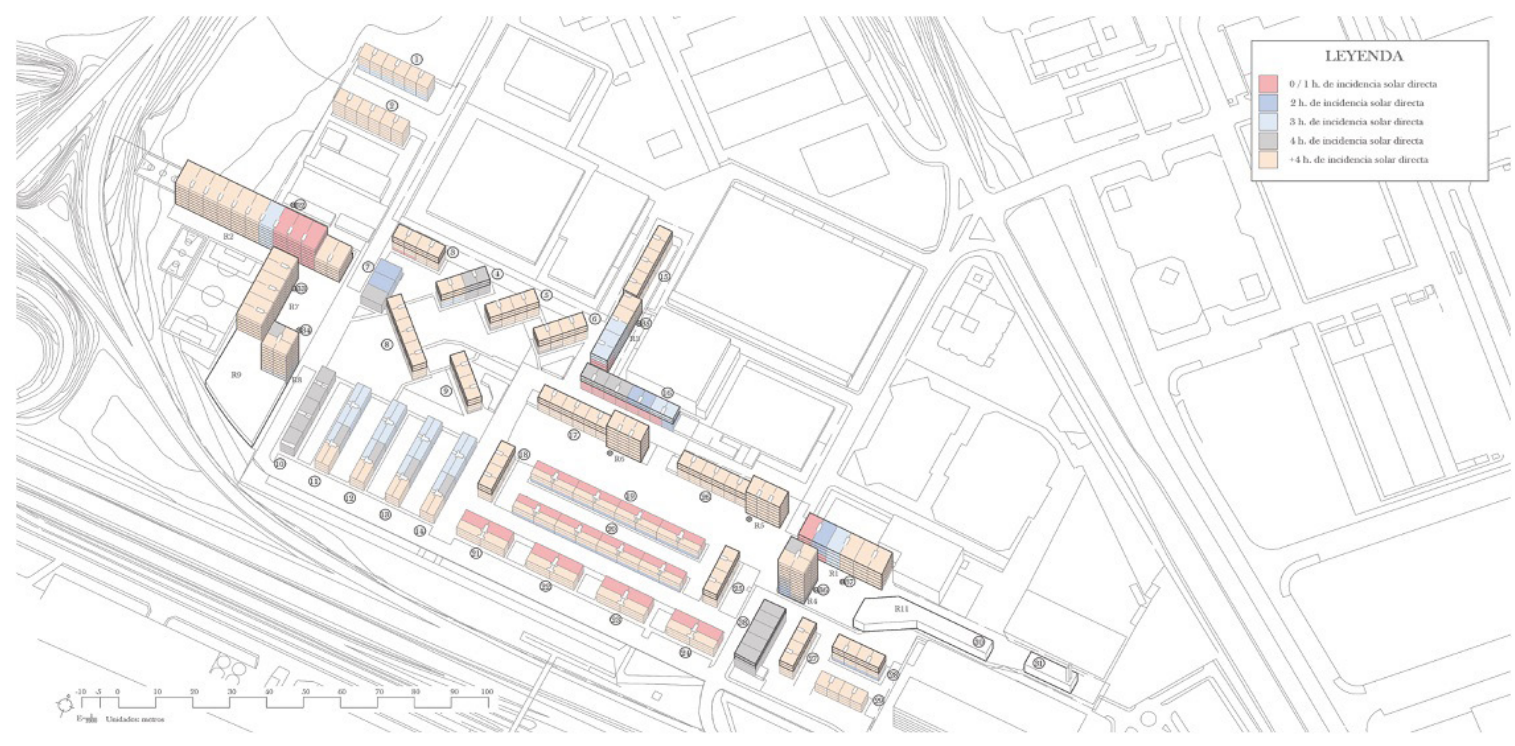

Fig. 7 Ejemplo de indicador mapificado para el barrio. Viviendas con menos de 4 horas solares en la suma de sus fachadas el 21 de diciembre. Fuente propia 
los resultados establecen que las emisiones de $\mathrm{CO} 2$ descenderían a $12,82 \mathrm{kgCO} 2 / \mathrm{m} 2$ año, ligeramente por debajo del escenario de la rehabilitación de los edificios existentes sin los aumentos de volumen del escenario 1 simulado. Se estima que el barrio produciría en la climatización de sus edificios una huella de carbono anual en servicio 884.080,02 kgCO2/año. En la modelización y análisis del potencial fotovoltaico del barrio regenerado con aumento de volumen y utilizando solo la mitad de la superficie de cubierta se estima una producción anual de energía de 2.571 .871 $\mathrm{kWh} / \mathrm{año.}$

¿Se puede financiar con la redensificación la regeneración del barrio?, Equilibrio de costes y beneficios. Gestión económica de la intervención

Con esos datos podemos evaluar el balance coste-beneficio de los tres escenarios, comprobando que la plusvalía obtenida es mayor en escenario1, donde, a pesar del coste mayor de la construcción de vivienda sobre edificios existente la estrategia de subir dos plantas en sólo algunos edificios ha permitido aumentar la cantidad de viviendas ofrecidas, (un $8 \%$ sobre el segundo escenario, pero un $28,6 \%$ sobre el tercero). Estas diferencias explican las variaciones de plusvalía.

A la vista del margen obtenido, resulta razonable hacerse la siguiente pregunta, ¿cuál sería la cantidad de viviendas nuevas que sería justificado construir en función de una reinversión en la rehabilitación de las existentes? Hemos intentado responderla estableciendo una ratio en función de los escenarios manejados.

Así, en el escenario 1 con la plusvalía que generan 145 viviendas estaríamos cubriendo

\begin{tabular}{|l|r|r|r|}
\hline & \multicolumn{1}{|l|}{ Escenario1 } & \multicolumn{1}{l|}{ Escenario2 } & \multicolumn{1}{|l|}{ Escenario3 } \\
\hline $\begin{array}{l}\text { no viv } \\
\text { rehab }\end{array}$ & 540 & 540 & 540 \\
\hline coste rehab & 21.956 .857 & 21.956 .857 & 21.956 .857 \\
\hline n viv libre + locales terciarios & 252 & 233 & 196 \\
\hline coste construcción & $39.907 .081,11$ & $41.782 .251,88$ & $37.952 .269,20$ \\
\hline ingresos viv libre & $78.013 .750,48$ & $74.181 .998,88$ & $72.406 .385,19$ \\
\hline
\end{tabular}

Fig. 8. Costes e ingresos estimados en cada uno de los escenarios. Fuente propia el coste de la rehabilitación y la propia construcción de estas viviendas nuevas, con gastos incluidos. Esto supone que podríamos rebajar el incremento de edificabilidad hasta un $58 \%$ de lo que incluye la propuesta, consiguiendo un barrio de 95 viviendas por ha. La ratio obtenida es que cada vivienda nueva casi paga la rehabilitación de cuatro viviendas existentes; para ser más precisos la ratio viv nueva/viv rehab, es de 0,27 . Dicho de otro modo, para pagar la rehabilitación se necesita incrementar en un cuarto la edificación existente. Si tenemos en cuenta los costes de urbanización que ascienden a poco más de 6 millones de euros, la ratio empeora hasta 0,32. En el escenario 2 no cambian mucho las cosas. Son 157 las viviendas nuevas necesarias para pagar la factura de la rehabilitación, lo que supone poder reducir la oferta de vivienda hasta un $68 \%$ de la considerada en este escenario, Esta reducción de edificabilidad llevaría a proponer un barrio de 97 viviendas / ha., con una ratio viv nueva/viv rehab de 0,29. Incluyendo los costes de urbanización estamos hablando de una ratio de 0,36

Por último, para el escenario 3, se han calculado 125 viviendas para cubrir los costes de rehabilitación, (un 64\% de las propuestas), lo que arroja una ratio de 0,23 , el más favorable de la serie. Es decir, que por cada vivienda nueva se rehabilitan cuatro viviendas y media. Casi con un quinto de aumento de la

\begin{tabular}{|l|c|c|c|}
\hline & Esc1 & Esc2 & Esc3 \\
\hline $\begin{array}{l}\text { no viv nuevas } \\
\text { cubren costes } \\
\text { rehab }\end{array}$ & 145 & 157 & 125 \\
\hline $\begin{array}{l}\text { \% viv nuevas } \\
\text { cubren costes } \\
\text { rehab }\end{array}$ & $58 \%$ & $68 \%$ & $64 \%$ \\
\hline $\begin{array}{l}\text { viv nueva } \\
\text { / viv rehab }\end{array}$ & 0,27 & 0,29 & 0,23 \\
\hline $\begin{array}{l}\text { no viv rehab que } \\
\text { paga cada vivienda } \\
\text { nueva }\end{array}$ & 4 & 4,5 & 3,5 \\
\hline
\end{tabular}

Fig. 9. Aumentos de edificabilidad estrictamente necesarios para cubrir los costes de rehabilitación 
edificación existente se para la rehabilitación de esas viviendas. Este escenario acabaría conformándose con una densidad de 93 viv/ ha. Con urbanización incluida la ratio sería de 0,28 .

\section{Conclusiones sobre sostenibilidad y viabilidad económica. Oportunidades de un modelo de intervención basado en cooperativas.}

Tras comparar la sostenibilidad dé cada escenario y su relación coste beneficio podríamos afirmar que se puede incrementar la densidad en el barrio del Aeropuerto siguiendo unos criterios de sostenibilidad aceptables, que consideren el aumento de la diversidad social y funcional del barrio, así como la mejora de su metabolismo urbano. Señalemos de modo sintético algunas otras conclusiones:

a) La rehabilitación puede ser costeada económicamente a partir de los incrementos de edificabilidad en un marco de sostenibilidad para el barrio. La redensificación del barrio puede acometerse de tal modo que se establece la siguiente relación: cada vivienda nueva costea la rehabilitación de cuatro viviendas existentes.

b) Las posibilidades de crecimiento pueden darse en edificios de nueva planta o con viviendas nuevas en edificios existentes. La discusión sobre qué escenario es mejor no arroja diferencias muy claras: si bien, desde el punto de vista de la viabilidad económica parece ligeramente más eficaz el escenario3, con sólo edificios de nueva planta que asumen el crecimiento del barrio, el escenario que se ha mostrado más eficaz en su comportamiento ambiental es el escenario 1

c) Los indicadores de sostenibilidad se han mostrado como una buena herramienta de control sobre propuestas de regeneración urbana. Tal y como han sido definidos, permiten evaluar decisiones de diseño urbano $\mathrm{y}$ arquitectónico sobre la edificación $\mathrm{y}$ el espacio público en los barrios a regenerar, así como establecer correcciones para poder situar las propuestas en un marco de sostenibilidad objetivable.

La cuestión que queda pendiente es cómo hacer el reparto de esta plusvalía generada. Una cuestión clave es la función de la administración pública en el proceso y que tipo de costes corren a su cargo. A partir de ahí entender que los recursos que se han generado en la regeneración del barrio también están a disposición de la propia administración local. Esto supone establecer un margen para la entrada de agentes económicos locales profesionalizados, sobre todo entendiendo que pueden. En una operación sin ánimo de lucro estos agentes pueden ser los propios vecinos organizados en cooperativas y asegurar así, su vinculación al proceso. Una posible organización de estas cooperativas la avanza la figura siguiente, en la que se combinan los

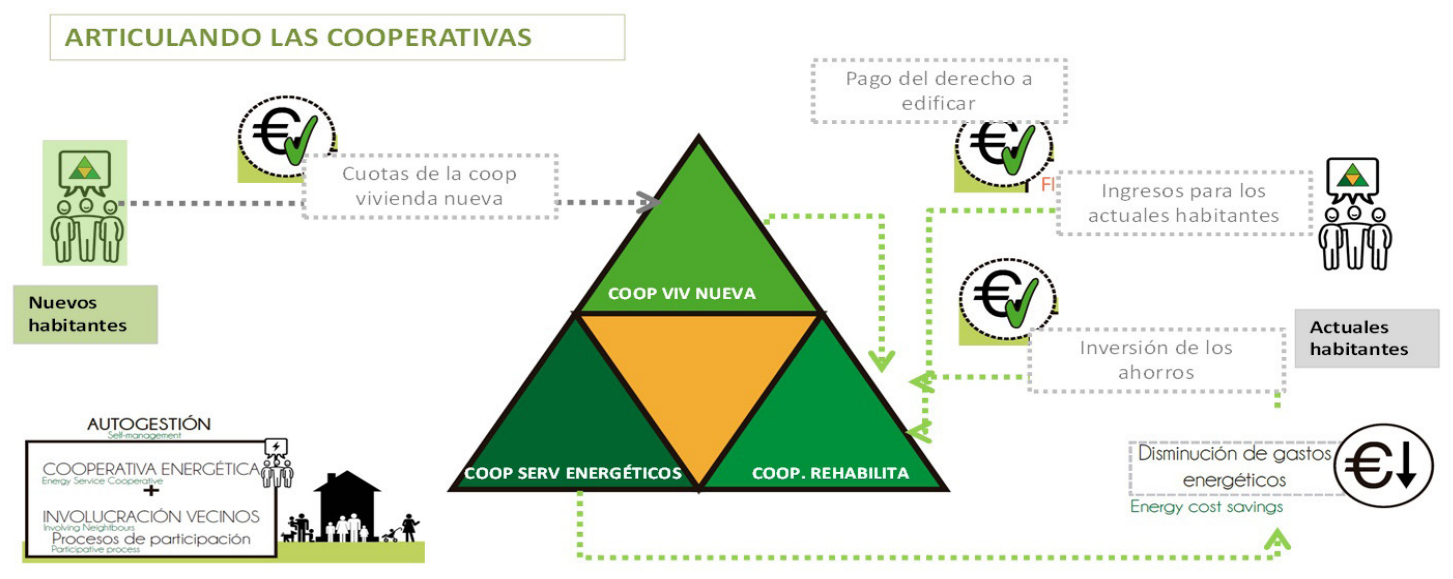

Fig. 10. Modelo de flujos de una cooperativa de regeneración urbana con tres tipos de cooperativas articuladas entre si. (González y Moreno S.,2015). 
flujos de capital que aportan cooperativas de vivienda nueva en el barrio, cooperativas de rehabilitación y alguna otra como al de servicios energéticos posible (González y Moreno, 2015).

\section{Notas}

1 Entendemos que aquí es de aplicación el concepto de Urban Growth Machine, término que desarrolla Harvey Molotch en su libro Urban Fortunes (1987) donde retoma el concepto acuñado en su clásico artículo de 1974 "The City as a Growth Machine". La idea es que la formalización de la ciudad, las decisiones sobre su morfología, física y social no parten de necesidades funcionales y de las relaciones interpersonales, sino de procesos sociales entre los que se encuentran las presiones de los agentes sociales sobre posiciones o lugares concretos.

2 Esta ley es entendida desde el ámbito local como una forma de invitar a la inversión privada, ya que la pública ni está ni se la espera bajo la austeridad obligada desde las políticas europeas. En muchos casos, la gobernanza de esta ley vista desde los ayuntamientos consiste en entender que el mercado se ocupa de todo que rehabilita "llave en mano" e involucra la financiación, convence a los vecinos por los beneficios inherentes de mejorar energéticamente sus viviendas y agrupa al resto de los agentes ante el pingüe negocio. Todo ello con a la simple supervisión de la autoridad local.

3 El origen de esta investigación es un encargo hecho por el Ayuntamiento de Madrid con el objetivo de aplicar indicadores de sostenibilidad a escala de barrio que supongan una fácil aplicación o control desde 1 labor de los técnicos municipales

4 Aplicado en la revisión del PGOU97 iniciada en 2010 (25 de noviembre de 2010). En nuestra opinión en buena medida estos indicadores siguen el modelo de los de Salvador rueda y escuela de ecología urbana de Barcelona.

5 Declaración de Toledo, Reunión Informal de Ministros. Unión Europea 2010.

6 En lo que se refiere a los agentes implicados en el proceso de regeneración urbana, la situación aislada del conjunto de la ciudad la vulnerabilidad del barrio viene proviene del alto envejecimiento de la población y de las condiciones de habitabilidad de las viviendas descritas antes. Sin embargo, es un barrio con una gran cohesión social que se articula a través de la asociación de vecinos, que ha demostrado ser muy operativa en impulsar y mantener el proceso de regeneración urbana incipiente. La apuesta del Ayuntamiento con una amplia financiación de los trabajos de rehabilitación de las viviendas ha permitido que los vecinos gestionen la elección del equipo de arquitectos que se está encargando de los proyectos de rehabilitación de todas las comunidades de propietarios. Un proceso de participación en marcha está permitiendo un diseño colaborativo de los espacios públicos, y muy especialmente los espacios entre bloques y su contacto con las viviendas en planta baja.

\section{Referencias}

BBVAServicio deEstudios Económicos (2008). "Proyecciones de vivienda terminada". En BBVA, Situación Inmobiliaria diciembre 2008.

Boorsboom-Van Beurden, Silvia; Doepel, Duzan and Tillie, Nico (2013) "Sustainable Densification and Greenification in the Inner City of Rotterdam" in CUPUM the International Conference on Computers in Urban Planning and Urban Management 13th edition, Utrecht, The Netherlands July 2013

Bosqued Alejandro., Gonzalez Francisco.J., Moreno Susana. (2017) "Social and Economic Management of Sustainable Neighborhoods Regeneration Projects". In: Álvarez Fernández R., Zubelzu S., Martínez R. (eds) Carbon Footprint and the Industrial Life Cycle. Green Energy and Technology. Springer, Cham

Claassens, Jip; Koomen, Eric and Rijken Bart (2018) "Analysing further urban densification: a case study for the Netherlands" in 57th ERSA Congress, August 29th-September 1st, Groningen, The Netherlands.

Cuchí, Albert; Sweatman, Peter. (2011). Una visión-país para el sector de la edificación 
en España: hoja de ruta para un nuevo sector de la vivienda. Grupo de Trabajo sobre Rehabilitación" GTR".

De Santiago, Eduardo. (2011). "Madrid,' ciudad única'. Pautas y lógicas espaciales recientes en la región madrileña". Urban, (12), 8-33.

De Schiller, Silvia; Evans, John Martin. (2000) "Urban climate and compact cities in developing countries" en Compact cities: sustainable urban forms for developing countries, , p. 117-124.

Fernandez Cristina.; Roch, Fernando. (2012) "La quiebra de la ciudad global y sus efectos en la morfología urbana. Madrid, bajo la lógica inmobiliaria de la acumulacióndesposesión", Urban n ${ }^{\circ}$ 3,2012, pags.45-63 ISSN 1138-0810

González, Francisco J. y Pérez, Ana. 2013 "Lo social en la regeneración urbana madrileña. Algunas notas sobre inmigración y gentrificación”. en Ed: CDU. Madrid: materia de debate 2003-2013. CDU. Madrid, Vol 4,pag 125-156. ISBN 978-84-616-92064

González, Francisco J. y Moreno Susana. (2015). "Pensar en cooperativas de regeneración urbana sostenible". Informes de la Construcción. Instituto de Ciencias de la Construcción Eduardo Torroja.CSIC. 67, pp.67-69. ISSN 0020-0883.,

Logan, John. R., y Molotch, Harvey. L. (2007). Urban fortunes: The political economy of place. Univ of California Press.

Molotch, Harvey.L. (1976). "The city as a growth machine: Toward a political economy of place". American journal of sociology, 82(2), 309-332.

Moreno, Susana., González, Francisco J. y Gómez Alberto. (2014). "La regeneración urbana en España : Revisión crítica para un estudio de caso de regeneración sostenible". Revista de Arquitectura, 19(27), Pág. 6-14. doi:10.5354/0719-5427.2014.33563

Naredo Jose.M (2004) "Naturaleza de la conurbación madrileña y sus tendencias actuales. Segunda parte. Anatomía y fisiología de la región urbana madrileña" en Urbanismo en el siglo XXI : una visión crítica : Bilbao, Madrid, Valencia, Barcelona / coord. por Jordi Borja i Sebastià, Zaida
Muxí Martínez, págs. 1001-119, ISBN 848301-740-7,

Newman, Peter. y Kenworthy Jeffrey R. (1989). Cities and Automobile Dependence: An International Sourcebook. Aldershot, UK:Gower Technical.

Roch, Fernando. (2004) "Naturaleza de la conurbación madrileña y sus tendencias actuales. Primera parte. Agentes sociales y tendencias urbanísticas" en Urbanismo en el siglo XXI : una visión crítica : Bilbao, Madrid, Valencia, Barcelona / coord. por Jordi Borja i Sebastià, Zaida Muxí Martínez, págs. 79-99, ISBN 84-8301-740-7

Swedish National Board of Housing, Building and Planning (2017) The urban density done right- Ideas on densification of cities and other communities, Karlskrona, Sweden ISBN print: 978-91-7563-466-1 